\title{
Studio preliminare di persistenza di batteri non fermentanti in un reparto di terapia intensiva e rianimazione
}

\author{
Alessandra Giordano, Serena Gerardi, Alberto Vanzetta, Paola Varesi, Carlo Mancini \\ Dipartimento Scienze e Sanità Pubblica, Sezione Microbiologia Università “La Sapienza”, Roma.
}

Key words: A. baumannii, P. aeruginosa, S. maltophilia, RAPD, susceptibility

Preliminary study of persistence on non- fermenting gram-negative bacteria in intensive care units

\section{SUMMARY}

A. baumannii, P. aeruginosa and S. maltophilia are important nosocomial pathogens, especially those collected from immunocomprimised patients and from intensive care units. In an attempt to define the epidemiology of non fermenting Gram negative bacteria, 87 P. aeruginosa, 50 A. baumannii and $22 \mathrm{~S}$. maltophilia, isolated from the respiratory districts and blood cultures of patients in the intensive care unit of our hospital, were studied to verify their antimicrobial susceptibility patterns and their diffusion. These strains were typed using ID-GNB card (bioMérieux) and antimicrobial susceptibility patterns were determined using AST-GN09 card VITEK-2 (bioMérieux). Molecular typing methods, such as RAPD have emerged as most efficient tools for strain discrimination, because antibiotic supsceptibility and other phenotyping methods are not reliable for epidemiological studies.

RAPD was performed on I5 P. aeruginosa, I2 S. maltophilia and II A. baumannii. The RAPD-PCR showed 7 different patterns in $P$. aeruginosa, 10 different patterns in S. maltophilia and the same profile in all strains of $A$. baumannii of patients admitted in intensive care units. Only two strains of A. baumannii of patients admitted in a thoracic surgical unit showed a different pattern in comparison to the other isolates.

\section{INTRODUZIONE}

I reparti di Terapia Intensiva e Rianimazione (RIA) sono un serbatoio di batteri Gram negativi non fermentanti molto spesso multiresistenti $(3,4,13,26)$; le specie più frequentemente isolate sono: P. aeruginosa, $S$. maltophilia e A. baumannii. Tali batteri hanno una distribuzione ambientale, ma possono essere considerati patogeni nei pazienti immunocompromessi $(8,9,11)$.

La colonizzazione batterica avviene principalmente a livello delle basse vie aeree e, spesso, le terapie risultano inefficaci in ragione della multiresistenza intrinseca o acquisita. La possibile infezione del tratto respiratorio con microrganismi multiresistenti può portare quindi al fallimento terapeutico con gravi ripercussioni sia sullo stato clinico del paziente, sia nella gestione dei pazienti ricoverati nello stesso reparto $(1,14,15$, 25). È noto che la valutazione epidemiologica contribuisce a facilitare il lavoro di prevenzione e controllo di tali infezioni (22). Nasce dunque la necessità di attivare una sorveglianza epidemiologica al fine di monitorare le infezioni ospedaliere $(5,10,17,18,21,24)$.

Profili di resistenza antibiotica, anche se identici, non sono indice di uguaglianza dei ceppi.
Esistono diverse metodiche bio-molecolari attendibili ai fini di discriminazioni clonali, della loro persistenza e della loro frequenza nel tempo che aiutano e facilitano gli studi epidemiologi: una di queste è la RAPD-PCR, rapida, con costi e tempi contenuti (23).

Tale metodica, basata sul principio del fingerprinting del DNA, è utilizzata per differenziare ceppi di specie diverse i diversi sierotipi all'interno di una specie e i vari sottotipi all'interno di un sierotipo; è utile, di conseguenza, per determinare se due isolati della stessa specie batterica sono epidemiologicamente correlati $(19,20)$. La metodica trae vantaggio da primer che si appaiano a monte di sequenze altamente ripetute e conservate, caratteristiche, per numero e dimensioni, dei singoli ceppi.

Il nostro lavoro è stato svolto nei primi sei mesi dell'anno 2005 presso il Laboratorio Analisi Microbiologiche B, Azienda Policlinico Umberto I Roma. È stato suddiviso in due fasi: uno studio fenotipico, al fine di valutare i profili batterici di resistenza agli antibiotici, ed uno studio genotipico, al fine di stabilire il clone o i cloni coinvolti negli episodi di colonizzazione e il grado di diffusione. Le fasi del lavoro sono state così suddivise: 
i) valutazione delle resistenze mediante AST GN09 card VITEK2 (bioMérieux) e/o metodica Kirby Bauer;

ii) applicazione della tecnica RAPD-PCR per il monitoraggio della persistenza di $P$. areuginosa, S. maltophilia e A. baumannii isolati da pazienti ricoverati presso la Terapia Intensiva e Rianimazione (RIA);

iii). raffronto dei profili genetici ottenuti, nel corso del tempo di indagine, sia nel singolo paziente che tra tutti i pazienti oggetto dell'indagine analitica.

\section{MATERIALI E METODI}

Sono stati isolati 87 P. aeruginosa, 22 S. maltophilia e 50 A. baumannii, di cui 48 provenienti da campioni respiratori di pazienti del reparto di Terapia Intensiva (RIA) e $2 \mathrm{~A}$. baumannii provenienti dal reparto di Chirurgia dello stesso Policlinico Umberto I. I ceppi isolati dai comuni terreni di coltura, sono stati identificati con IDGNB-card Vitek2 (bioMérieux). La valutazione delle resistenze batteriche è stata effettuata mediante AST-GN09 card Vitek2 (bioMérieux) contenente i seguenti antibiotici: amikacina a 8,16 , e $64 \mu \mathrm{g} / \mathrm{ml}$; ampicillina a 4,8 e $32 \mu \mathrm{g} / \mathrm{ml}$; ampicillina/sulbactam a $4 / 2,16 / 8$, e $32 / 16 \mu \mathrm{g} / \mathrm{ml}$, aztreonam a 2,8 e $32 \mu \mathrm{g} / \mathrm{ml}$; cefazolina a 4,16 , e 64 $\mu \mathrm{g} / \mathrm{ml}$; cefepime a $2,8,16$, e $32 \mu \mathrm{g} / \mathrm{ml}$; cefotetan a 2,8 , e $32 \mu \mathrm{g} / \mathrm{ml}$; ceftazidime a $1,2,8$, e $32 \mu \mathrm{g} / \mathrm{ml}$; ceftriaxone a $1,2,8$, e $32 \mu \mathrm{g} / \mathrm{ml}$; cefuroxime a 2,8 e $32 \mu \mathrm{g} / \mathrm{ml}$; ciprofloxacina a $0.5,2$, e $4 \mu \mathrm{g} / \mathrm{ml}$; gentamicina a 4,16 , e $32 \mu \mathrm{g} / \mathrm{ml}$; imipenem a 2,4 , e 16 $\mu \mathrm{g} / \mathrm{ml}$; levofloxacina a $0.5,4$, e $8 \mu \mathrm{g} / \mathrm{ml}$; meropenem a $0.5,4$ e $16 \mu \mathrm{g} / \mathrm{ml}$; nitrofurantoina a 16,32 e $64 \mu \mathrm{g} / \mathrm{ml}$; piperacillina a 4,16 e $64 \mu \mathrm{g} / \mathrm{ml}$; piperacillina/tazobactam a $4 / 4,16 / 4$, e $128 / 4 \mu \mathrm{g} / \mathrm{ml}$; tobramicina a 8,16 , e $64 \mu \mathrm{g} / \mathrm{ml}$, trimethoprim/sulfamethoxazole a $0.5 / 9.5,2 / 38$ e $16 / 304 \mu \mathrm{g} / \mathrm{ml}$. Per A. baumannii è stata inoltre saggiata la colistina (Taxo bioMérieux $25 \mu \mathrm{g}$ ) mediante metodica Kirby Bauer. Per tutti i ceppi di S. maltophilia, la validazione del Vitek 2 è stata per il solo trimethoprinsulfametossazolo, unico antibiotico validato dall'F.D.A. nei sistemi automatici. I ceppi sono stati successivamente conservati in Microbank (Pro-Lab Diagnostic Biolife) a $-35^{\circ} \mathrm{C}$.

L'estrazione del DNA dei ceppi batterici è stata eseguita, dopo incubazione in BHI (Becton Dickinson) overnight a $37^{\circ} \mathrm{C}$, utilizzando il Kit Wizard Genomic (Promega) secondo le istruzioni della casa produttrice. La quantizzazione è stata eseguita mediante lo spettrofotometro GeneQuant II (Pharmacia Biotech) ad un'assorbanza compresa tra i valori di 0,05 e 2 DO.

L'amplificazione è stata eseguita mediante un Thermal Cycler Progene (Techne) programmato in modo differente a seconda della specie batterica in esame ed i primers utilizzati sono stati $\mathrm{i}$ seguenti: $P$. aeruginosa- primer 10514 5'TGGTGGCCTCGAGCAAGAGAACGGAG -3' $(6,7) ; \quad S$. maltophilia- primer 270 5'TGCGCGCGGG-3' (16); A. baumannii- primer ACI 5' -GCTTGTGAAC-3' (2).

La corsa elettroforetica su gel di agarosio preparato all' $1 \%$ per $A$. baumannii e all' $1,5 \%$ per $P$. aeruginosa e $S$. maltophilia è stata condotta ad un voltaggio di $150 \mathrm{~V}$. Al gel sono stati aggiunti $0.5 \mu 1 / \mathrm{ml}$ di bromuro di etidio, cosi che i prodotti della PCR potessero essere visualizzati e fotografati su transilluminatore U.V. (GENENCO).

Gli isolati differenti per due o più bande prominenti sono stati considerati sufficientemente divergenti per essere classificati come nuovo genotipo. I profili differenti per una sola banda prominente o una o due bande deboli sono stati considerati sottotipi di un comune ceppo epidemico (23). La valutazione dell'analisi genomica su $P$. aeruginosa, $A$. baumannii ed $S$. maltophilia, mediante RAPD-PCR, è stata ripetuta, per confermare la ripetitività del dato, eseguendo tre volte le amplificazioni.

\section{RISULTATI}

La valutazione fenotipica ha evidenziato spesso multiresistenze sia per A. baumannii che per P. aeruginosa con preoccupanti resistenze nei confronti degli aminoglicosidi e dei carbapenemici. Tutti i ceppi di A. baumannii sono risultati tuttavia sensibili alla colistina. S. maltophilia è risultato essere sempre sensibile al trimethoprin-sulfametossazolo.

Per quanto riguarda lo studio genotipico dei profili i risultati di una selezione di 15 ceppi di $P$. aeruginosa, isolati da pazienti diversi, hanno evidenziato la presenza di 7 profili genetici differenti, indicando variabilità e non persistenza di un unico clone. Osservando la figura I si può notare come le corsie 2-5-10-11-12 presentano lo stesso profilo, come anche le corsie 3-4, 7-14-16 e 8-13. Nei 12 ceppi di S. maltophilia selezionati, sono stati evidenziati 10 profili genotipici differenti e lo stesso profilo è stato evidenziato su tre ceppi provenienti da campioni diversi di uno stesso paziente (corsie 4-6-9) (figura II). Degli 11 ceppi di $A$. baumannii selezionati, 9 ceppi provenienti da pazienti diversi ricoverati nel reparto di RIA hanno mostrato lo stesso profilo, mentre gli altri 2 ceppi (corsia 7-11), provenienti dal reparto di chirurgia toracica e dallo stesso paziente, hanno presentato lo stesso profilo genotipico, ma diverso dagli isolati della RIA. Ciò dimostra la persistenza di un ceppo endemico, diffuso nel reparto di RIA, confermando un'epidemia di fonte comune (figura III). 


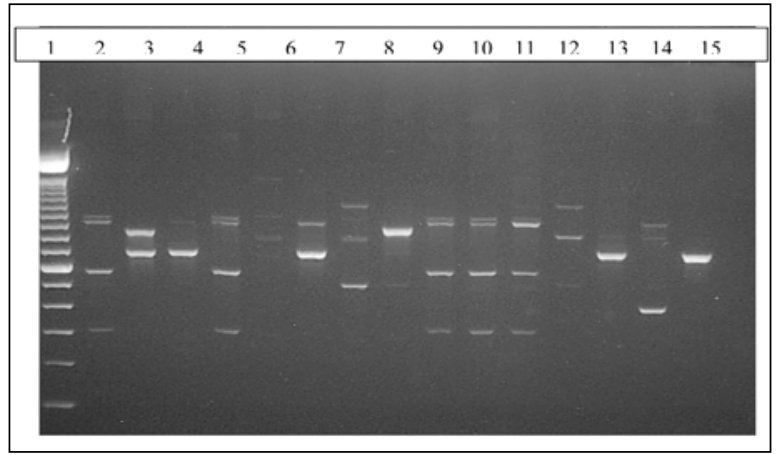

Figura I.

Analisi dei profili genetici di P.aeruginosa mediante RAPD-PCR. (Corsia I standard 100pb, corsie 2-I6 amplificati batterici, corsia I 7 controllo negativo)

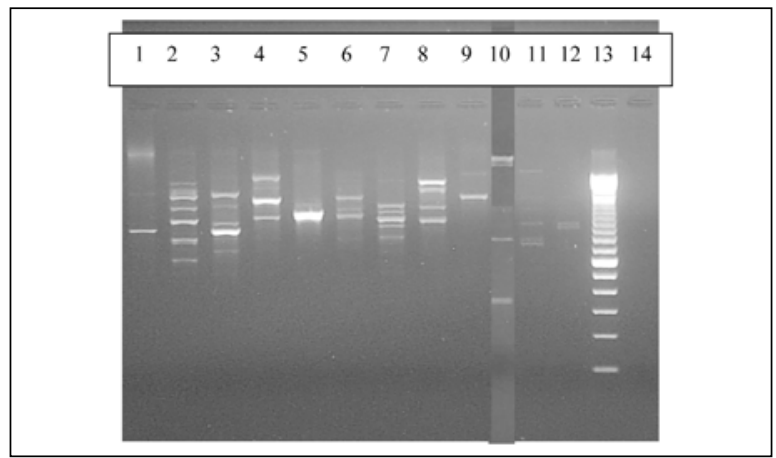

Figura II.

Analisi dei profili genetici di S.maltophilia mediante RAPD-PCR. (Corsia I-12 amplificati batterici, corsia I3 standard 100pb, 14 controllo negativo)

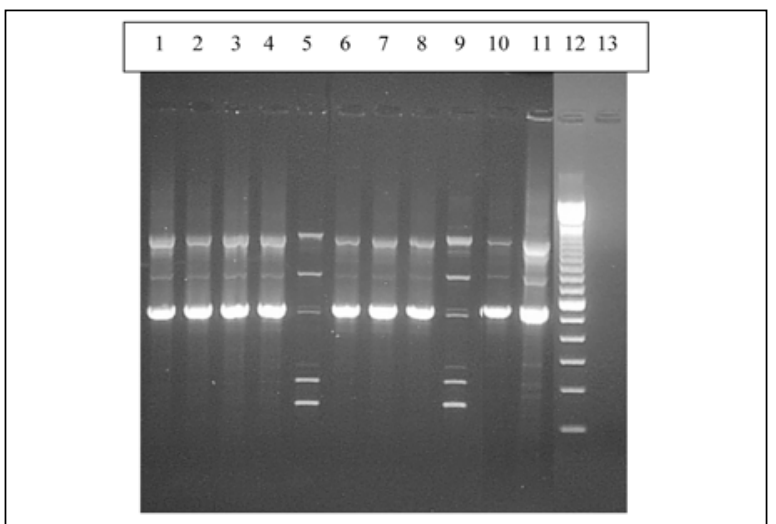

Figura III.

Analisi dei profili genetici di A.baumannii mediante RAPD-PCR. (Corsie I-I I amplificati batterici, corsia I2 standard 100pb, corsia 13 controllo negativo)

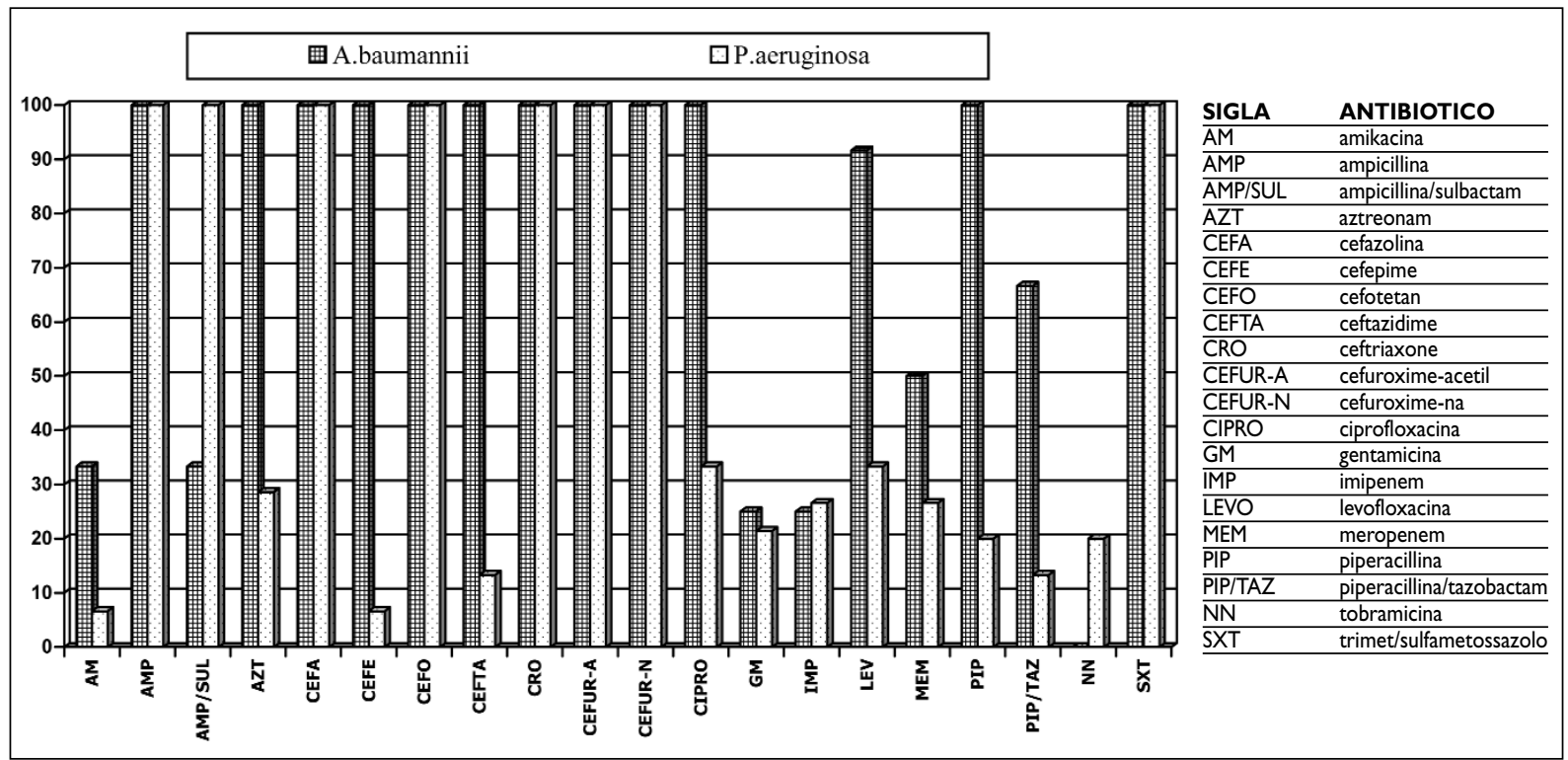

Grafico I. Percentuali di resistenze batteriche agli antibiotici.

\section{CONCLUSIONI}

L'aumento del ritrovamento dei batteri oggetto di indagine è stato probabilmente innescato dall'uso di terapie antibiotiche a largo spettro e/o prolun- gate e dalla maggior frequenza di malattie polmonari croniche (11-14). La pulsed-field gel electrophoresis (PFGE) è considerata il metodo goldstandard per la tipizzazione genotipica, tuttavia la 
differenza di sensibilità si scontra con svantaggi pratici, quali i tempi di risposta più lunghi e i costi superiori. L'analisi mediante RAPD-PCR può essere usata come alternativa o complementare per la tipizzazione genotipica nel corso di colonizzazioni e/o infezioni; pertanto, a nostro avviso, è uno strumento fondamentale nel controllo e nella prevenzione delle infezioni ospedaliere.

\section{BIBLIOGRAFIA}

1. Ayan M, Durmaz R, Aktas E, et al. Bacteriological, clinical and epidemiological characteristics of hospital-acquired Acinetobacter baumannii infection in a teaching hospital. J Hosp Infect 2003; 54(1): 39-45.

2. Carr E, Eason H, Feng S, et al. RAPD-PCR typing of Acinetobacter isolates fromactivated sludge systems designed to remove phosphorus microbiologically. J Appl Microbiol 2001; 90: 309-19.

3. Canton R, Valdezate S, Vindel A, et al. Antimicrobial susceptibility profile of melecular typed cystic fibrosis $S$. maltophilia isolates and differences with noncystic fibrosis isolates. Ped Pulmon 2003; 35: 99-107.

4. Cisneros JM, Reyes MJ, Pachón J, et al. Bacteremia due to Acinetobacter baumannii: epidemiology, clinical findings, and prognostic features. Clin Infect Dis 1996; 22: 1026-32.

5. Crispino M, Boccia MC, Bagattini M, et al. Molecular epidemiology of $S$. maltophilia in an university hospital. J Hosp Infect 2002; 52: 88-92.

6. Kersulyte D, Struelens MJ, Deplano A, et al. Comparison of arbitrary primed PCR and macrorestriction (Pulsed-Field Gel Electrophoresis) typing of Pseudomonas aeruginosa strains from Cystic fibrosis patients. J Clin Microbiol 1995; 33(8): 2216-9.

7. Mahenthiralingam E, Campbell ME, Foster J, et al. Random amplified polymorphic DNA typing of Pseudomonas aeruginosa isolates recovered frompatients with cystic fibrosis. J Clin Microbiol 1996; 34: 1129-35.

8. Denton M, Kevin GK. Microbiological and clinical aspects of infection associated with S. maltophilia. Clin Microbiol Rev 1998; 11: 57-80.

9. Elting LS, Khardori N, Bodey GP, et al. Nosocomial infection caused by $X$. maltophilia: a case-control study of predisposing factors Infect Control Hosp Epidemiol 1990; 11: 134-8.

10. Fadda G, Spanu T, Ardito F, et al. Antimicrobial resistance among non-fermentative Gram-negative bacilli isolated from the respiratory tracts of Italian inpatients: a 3-year surveillance study by the Italian Epidemiological Survey. Italian Epidemiological Observatory. Int J Antimicrob Agents 2004; 23(3): 254-61.

11. Forster DH, Daschner FD. Acinetobacter spp as nosocomial pathogens. Eur J Clin Microbiol Infect Dis 1998; 17: 73-7.

12. Fridkin SK. Increasing prevalence of antimicrobial resistance in intensive care units. Crit Care Med 2001; 29: N64-N68.

13. Garrison MW, Anderson DE, Campebell DM, et al. $S$. maltophilia: emergence of multidrug.-resistant strains during therapy and in an in vitro pharmacodynamic chamber model. Antimicrob Agents Chemother 1996; 40: 2859-64.

14. Hanes SD, Demirkan K, Tolley E, et al. Risk factors for late-onset nosocomial pneumonia caused by $S$. maltophilia in critically ill trauma patients. Clin Infect Dis 2002; 35: 228-35.

15. Kanafani ZA, Kara L, Hayek S, et al. Ventilator-associated pneumonia at a tertiary-care center in a developing country: incidence, microbiology, and susceptibility patterns of isolated microorganisms. Infect Control Hosp Epidemiol 2003; 24(11): 864-9.

16. Krzewinski JW, Nguyen CD, Foster JM, et al. Use of random amplified polymorphic DNA PCR to examine epidemiology of S. maltophilia and Achromobacter (Alcaligenens) xylosoxidans from patients with cystic fibrosis. J Clin Microbiol 2001; 39: 3597-602.

17. Laing FPY, Ramotar K, Read RR, et al. Molecular epidemiology of $S$. maltophilia colonization and infection in the hospital environment. J Clin Microbiol 1995; 33: 513-8.

18. Marroni M, Pasquarella C, Agodi A, et al. Clonal spread of Acinetobacter baumannii in a general intensive care unit. Ann Ig 2004; 16(1-2): 95-102.

19. Ortiz-Herrera M, Geronimo-Gallegos A, Cuevas Schacht F, et al. RAPD-PCR characterization of Pseudomonas aeruginosa strains obtained from cystic fibrosis patients. Salud Publica Mex 2004; 46(2): 149-57.

20. Schutze GE, Gilliam CH, Jin S, et al. Use of DNA fingerprinting in decision making for considering closure of neonatal intensive care units because of Pseudomonas aeruginosa bloodstream infections. Pediatr Infect Dis J 2004; 23(2): 110-4.

21. Struelens MJ, Byl B, Vincent JL Antibiotic policy: a tool for controlling resistance of hospital pathogens. 1999; 5 Suppl 1: S19-S24.

22. Struelens MJ, and the Members of the European Study Group on Epidemiological Markers (ESGEM), of the European Society for Clinical Microbiology and Infectious Diseases (ESCMID) 1996. Consensus guidelines for appropriate use and evaluation of microbial epidemiologic typing systems. Clin Microbiol Infect 1996; 2: 2-11.

23. Suenga E, Nakamura H. Multiple-locus variable number tandem repeats analysis for genetic fingerprinting of pathogenic bacteria. Electrophoresis 2005; 26(13): 2567-82.

24. Valdezade S, Vindel A, Martìn-Dàvila P, et al. High genetic diversit among $S$. maltophilia strains despite their originating at single hospital. J Clin Microbiol 2004; 42: 693-9

25. Villers D, Espaze E, Coste-Burel M, et al. Nosocomial Acinetobacter baumannii infections: microbiological and clinical epidemiology. Ann Int Med 1998; 129: 182-9.

26. Zhang L, Li XZ, Poole K. Multiple antibiotic resistance in S. maltophilia: involvement of a multidrug efflux system. Antimicrob Agents Chemother 2000; 44: 287-93.
Alessandra Giordano
Dipartimento Scienze e Sanità Pubblica
Sezione di Microbiologia
Università "La Sapienza"
P.le Aldo Moro, 5 - 00185 Roma
E-mail: alessandra.giordano@uniroma1.it 